\title{
ON RECURRENT SOLAR ACTIVITY
}

\author{
V. GAIZAUSKAS \\ Herzberg Institute of Astrophysics \\ National Research Council of Canada \\ Ottawa, Canada K1A 0R6
}

\begin{abstract}
Recurrent solar activity on a time-scale of the Sun's rotation rate has for a long time been attributed to quasi-periodic disturbances in the solar interior. The following brief survey discusses recent attempts to come to grips with the Sun's episodic activity.
\end{abstract}

\section{INTRODUCTION}

The equatorward drift of the mean latitude of sunspots as a solar cycle advances has long been recognized as a basic ingredient for modeling the 11year cycle of activity. The search for a comparable systematic dependance on solar longitude has not yet resulted in a consensus for representing recurrent activity in models of the solar cycle.

The existence of recurrent solar activity at preferred longitudes is not in doubt. Carrington (1855) already drew attention to this tendency in his observations of $\mathbf{1 8 5 4}$ for activity to recur preferentially at some longitudes while completely avoiding others. He hypothesized the 'revolution of an inner body, on which may be situated the volcanic centres of eruption, in a shorter period than the envelope on which we see the effects in the form of ruptures of continuity'. A tendency for new regions to emerge near existing ones was known to the compilers of the Greenwich Photoheliographic Results who had the foresight to distinguish between 'recurrent spots' and 'revivals'.

Interest in recurrent solar activity has broadened since those early days to include a variety of phenomena. Two general approaches are taken to investigate the implications of recurrence: one involves harmonic or statistical analysis of single, time-dependant parameters (e.g. sunspot number, area, flare rates); the other examines spatial distributions (e.g. active-region positions, maps of magnetic flux). Harmonic analysis of rich sets of solar data bears directly on solar rotation and leads to inferences about rotational periods for 
the interior. The additional parameter supplied from spatial distributions alters our perspective on these problems. In particular, the 'revival' aspect of recurrent activity has been revealed as a tendency for active regions to form intermittently over long intervals of many rotations in tightly-defined clusters or 'nests'. The variety of interpretations issuing from these different approaches emphasizes the subtle character of the mechanism which sustains the recurrences. A selective sampling rather than a comprehensive survey follows of both approaches to the riddle of recurrent activity.

\section{HARMONIC OR STATISTICAL ANALYSIS OF SINGLE PARAMETERS}

\subsection{Sunspot Number}

Short-term periods were sought in a 122-year segment of the Wolf sunspot record by Knight, Schatten and Sturrock (1979) in an effort to corroborate evidence from oblateness measurements for a rapidly rotating solar core (Dicke 1976). Their least-squares procedure yielded a statistically significant peak at a period near 12 days in reasonable accord with Dicke's result.

Bogart (1982) performed an autocorrelation analysis of a 128-year record of daily sunspot numbers and found: (i) that recurrences with a period of 27 days persisted to about 10 solar rotations; (ii) that the strength and persistence of recurrent activity varied considerably from one 11-year solar cycle to another; (iii) that the rotational period inferred from the spacings produced by recurrent activity of successive maxima in the correlograms varied greatly $(\sim 2$ days); (iv) a peak at a period of 13.6 days in the power spectra, corresponding to the first harmonic of the dominant frequency of recurrence, thus implying a trend towards the formation of pairs of 'active longitudes' spaced $180^{\circ}$ apart.

Wolff (1983) postulated $g$-mode oscillations of the entire Sun to be responsible for the episodic nature of strong fluctuations in solar activity and for the existence of large-scale rigid patterns on a differentially rotating surface (Section 3). He therefore searched the Fourier spectrum of a 229-year sample of sunspot numbers for narrow spectral lines corresponding to $g$-mode harmonics at periods less than 2 years. He found precise agreement with theory for seven lines identified with angular harmonics $l$ of order 4 or greater.

Sunspot numbers provided part of the data base for a renewed search by Sturrock and Bai (1992) for an internal solar 'clock' to account for a complex of solar periodicities near the 154-day period first discovered in flare activity (Section 2.2). By Fourier-analysing long records of sunspot numbers, areas, and flares, they identified frequencies consistent with harmonics and sidebands of a hypothetical oscillator (driven by an unspecified process) rotating obliquely with respect to the ecliptic. The notion that the Sun's radiative core and convective envelope rotate about different axes was subsequently tested by 
Goode and Thompson (1992) against helioseismic data with negative results.

\subsection{Solar Flares}

Time-sequences of strong flares have for a long time been analysed for periodic behaviour, mainly with the aim of forecasting proton flares (as reviewed by Svestka 1976 ). These events are rooted in active regions of high magnetic complexity whose appearances are restricted to sporadic episodes of intense activity during any solar cycle. These special flare episodes have come under increasing scrutiny with the discovery by Rieger et al. (1984) of a 154day periodicity in occurrences of flares emitting energy in bands $>300 \mathrm{keV}$.

Bai (1988) examined recurrent flare activity in three solar cycles and found that superactive regions with major flares appear more frequently in restricted areas on the Sun which he called 'hot spots'. These special areas are active intermittently and can be traced by their periods of recurrence from one solar cycle to the next. He initially attributed hot spots to long-lived subsurface activity which rotates rigidly but at slightly different rates in the northern and southern hemispheres. With the detection by power spectrum analysis of additional periodicities in the record of major flares (Bai and Sturrock 1991; Bai 1992), the Stanford workers have focused upon the existence of an internal solar 'clock' which promotes surface activity at a complex of periodicities, not just one (Section 2.1).

\subsection{Sunspot Areas}

The record of sunspot areas compiled in the Greenwich Photoheliographic Results has also been analysed for periodic behaviour over many solar cycles, especially in the range favoured by recurrent flare activity (Carbonell and Ballester 1992, and references therein). Applying a periodogram procedure designed specifically for analysing time series with unevenly sampled data to cycles $12-21$, Carbonell and Ballester found periodicities in the range 150 $160 \mathrm{~d}$ to be significant in some solar cycles but not in others. A sliding window analysis revealed, within most of the selected solar cycles, intervals wherein a strong periodicity appears at $155.6 \mathrm{~d}$. This feature, related to the size of active regions, is consistent with the intermittent behaviour already apparent in the time series of strong flares.

These recent analyses of time-sequences of daily solar indices reaffirm the existence of recurrent phenomena and expose their intermittency. The multitude of periodicities reported so far, if genuine and not artifacts of the analytical procedures, indicates a complex oscillatory process for regulating solar activity. We turn next to regularities in the spatial distribution of solar activity for further insight into recurrent phenomena. 


\section{ANALYSES OF SPATIAL DISTRIBUTIONS OE SOLAR ACTIVITY}

\subsection{Photospheric Magnetic Flux}

The search for large-scale regularities in the line-of-sight component of photospheric magnetic flux began as soon as long sequences of synoptic maps plotted for individual Carrington rotations became available from magnetographic measurements. In their pioneering study of a $41 / 2$-yr interval of Mt. Wilson data, Bumba and Howard (1965) noted organized patterns which they attributed to the expansion, weakening, and stretching by differential rotation of the magnetic flux of old active regions. The interactions of dispersing with neighbouring fields and the continuing development of new regions were cited as factors in the process of organization. They also drew attention to the tendency for active regions to cluster in 'complexes of activity', an action which persisted up to 8 rotations in their sample. It was claimed that complexes spread systematically in both latitude and longitude and eventually covered a large longitudinal range over both the northern and southern active belts. In a subsequent study of an 8-yr interval of activity, Bumba and Howard (1969) found stronger evidence for large-scale regularities, persisting for many rotations, in the alternation of magnetic polarity with longitude. The background features with strong fields were also shown to resist the shearing effects of differential rotation.

The tendency for active regions to cluster in complexes of activity was confirmed for a different epoch of solar activity by Gaizauskas et al. (1983). Their synoptic magnetic maps were obtained at higher spatial resolution and with greater dynamic range than those of Bumba and Howard. They showed that the tendency for bipolar regions to cluster persists throughout the lifetime of an activity complex ( 3 to 6 rotations in their sample) because new active regions keep emerging to replace vanishing older ones inside the same complex. Flux does not keep accumulating during this process; it grows rapidly at the birth of a complex, remains roughly steady through its lifetime, and then declines abruptly. The greater part of the flux from individual bipolar regions must therefore vanish locally rather than disperse by a process of turbulent diffusion beyond the boundaries of the activity complex as envisioned by Leighton (1964). The spreading found by Bumba and Howard (1965) of complexes in latitude across the equator is not supported by highresolution data. During their active lifetimes, activity complexes are limited to a small range in latitude.

\subsection{Sunspot Positions}

More objective procedures applied to different compilations of data confirm in more rigorous fashion the tendency for active regions to form in clusters. For their graphical-statistical analysis of the positions of recurrent groups of sunspots, Castenmiller, Zwaan, and van der Zalm (1986) coined the term 
'sunspot nests' to evoke the spatio-temporal nature of the clustering phenomenon. Their study established the equivalence of 'activity complexes' and 'nests' in aspects such as: (i) appearing double with parallel, diverging, or merging branches, (ii) rotating about the Sun at a steady rate distinctive to each nest/complex. It also extended the basic properties of the phenomenon to matters such as compactness (areas no larger than a medium-large active region), lifetimes up to about 15 rotations (allowing for gaps up to 2.5 rotations), and prevalence (at least $30 \%$ of all sunspot groups). A standard mathematical technique of cluster analysis was applied by Brouwer and Zwaan (1990) to the same data set examined by Castenmiller, Zwaan and van der Zalm (1986). Results of their entirely objective procedures for identifying clusters are in accord with the earlier findings. Their method brings out the smallest scales in the clustering tendency: the centers of gravity of successive nests fall within an area much smaller than a medium-large sunspot group. But there is also a strong tendency for nests to cluster again in still larger nests: more than a third of the compact nests are components of larger ones. The existence of several levels of clustering was established by Petrovay and Abuzeid (1991) in their analysis by an entirely different clustering algorithm of sunspot positions for all of Cycle 20. The cycle-averaged rotational rates of the clusters detected by their procedure exhibit strongly reduced differential rotation compared to conventional tracers.

\subsection{Formation of Nests During a Solar Cycle}

Figure 1 illustrates some of the properties of nests discussed above. The figure assembles data, separately for each hemisphere, for 100 Carrington rotations during Cycle 21 . It was constructed by stacking $30^{\circ}$-wide latitudinal strips, one per Carrington rotation, extracted from successive synoptic maps of the photospheric magnetic flux measured at the National Solar Observatory (Kitt Peak). White patches denote positive (outward-directed) magnetic fields, black patches denote negative polarity. Longitude $(\mathrm{L})$ increases from left (east) to right and repeats for one-third rotation; time (t) advances downward. In the $\sim 7.5 \mathrm{yr}$ period sampled here the activity belts migrate equatorward from the latitude of $40^{\circ}$ used as the maximum of the strips at the beginning of the sample. The central latitude of each $30^{\circ}$ strip was therefore shifted according to Spörer's Law before the data were extracted from the full synoptic maps. The maps are uncompensated for the differential rotation of the Sun; their frame of reference rotates rigidly at the Carrington rate.

Nests form where activity keeps reviving on successive rotations due to injections of fresh magnetic flux as new bipolar regions. In the L-t plane they appear as tracks of different lengths, spacings, slopes, which are sometimes double (e.g. both hemispheres, just below the data gap in Rotation 1661).

The degree of organization of the tracks changes with phase of the solar cycle - widely spaced in longitude during the onset (1977-78) and the decay 


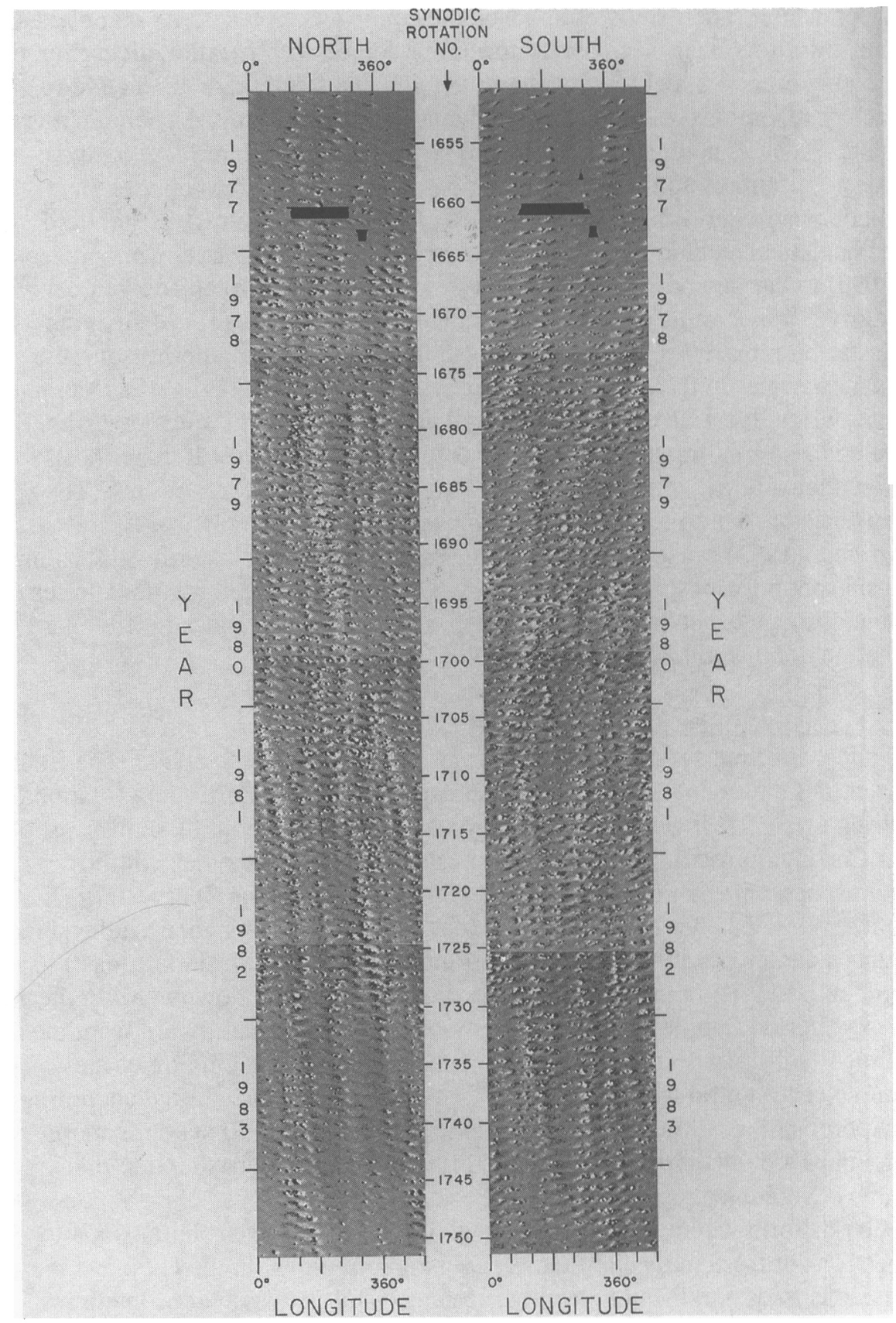

Fig. 1. Active belts in both hemispheres of the Sun for 100 Carrington rotations during Cycle 21. Construction is described in Section 3.3. 
(1982-83), but more closely packed (1979, $\mathrm{N}$ hemisphere) or even chaotic (1979-80, S hemisphere) during the maximum phase. Even during highly active periods there can be longitudinal zones over $90^{\circ}$ wide which are relatively free of activity (centered near $0^{\circ}, \mathrm{N}$ hemisphere, early in 1980 , and near $190^{\circ}, \mathrm{S}$ hemisphere, in mid-1981). Some nests are fully active for an entire year $\left(1982, \mathrm{~N}\right.$ hemisphere, near $\mathrm{L}=330^{\circ}$ ); others fade out after a brief period of recurrent activity only to revive a few rotations later for several more lively rotations $\left(1982, \mathrm{~N}\right.$ hemisphere, near $\mathrm{L}=120^{\circ}$ ). Indeed without specifying a rigorous criterion for associating recurrent activity in successive rotations as Brouwer and Zwaan (1990) did, it is difficult to link the track of one nest with another in a convincing way. The lifetimes of some nests may thus be greater than appears in a superficial glance at Figure 1.

A nest rotating around the Sun at the Carrington synodic period of 27.275 d produces a vertical track (e.g. $\mathrm{N}$ hemisphere, early 1984 at $\mathrm{L}=110^{\circ} ; \mathrm{S}$ hemisphere, in $1977-8$ at $\mathrm{L}=190^{\circ}$, early 1983 at $\mathrm{L}=180^{\circ}$ ). Nests rotating faster than the Carrington rate produce tracks sloping down to the west (right); nests lagging behind the Carrington rate produce tracks sloping down to the east (left). In the $\mathrm{N}$ hemisphere, nests with leading and lagging rates are both present during 1977-79. Leading rates dominate thereafter with a few exceptions. Leading and lagging rates are both found in the $S$ hemisphere during 1977-80. Again leading rates dominate thereafter. These subjective impressions, it should be cautioned, have yet to be quantified; they are strongly influenced by the strength of the magnetic flux in a particular nest.

\section{THEORETICAL IMPLICATIONS}

Although one may be tempted to pick out one or two tracks in Figure 1 for identification with the long-lived 'hot spots' of Bai (1988), it is clear that similar recurrent activity at weaker levels goes on simultaneously at many other places. The results of Brouwer and Zwaan (1990) and of Petrovay and Abuzeid (1991) tell us something that the harmonic/statistical analyses of solar indices cannot. And that is: the generator for the recurrent activity is highly localized and may at times be a superposition of multiple excitors. We can speculate that 'hot spots' arise in locales where multiple excitors are superposed while single excitors result in normal nests.

The injection of intense magnetic fields in bursts at a few localized sites has been modeled by Parker (1987) as a thermal relaxation oscillator. He proposes that an intense azimuthal magnetic field $(>3 \mathrm{kG})$ is pressed down into the lower convective zone by its thermal shadow. The accumulation of heat beneath the field causes the gas heated below to penetrate intermittently through the field, sending thermal plumes of gas with entrained fields to the surface at irregular intervals $\sim 1$ week. Wentzel and Seiden (1992) reproduced 
the quasi-regular distribution of active regions in clusters by using percolation theory. Their model bundles complicated MHD and turbulent processes into 2 dimensionless parameters: one expresses the probability for the release and rise of one flux tube to stimulate the subsequent release and rise of its neighbours; the other measures the lifetime of flux once it arrives at the surface.

So far these studies agree on one point: recurrent activity in both space and time is a basic property of the Sun. Consensus on its origin remains elusive.

\section{ACKNOWLEDGEMENTS}

I am indebted to J.W. Harvey for creating Figure 1 and for permission to publish it. The hospitality of the Colloquium organizers is deeply appreciated.

\section{REFERENCES}

Bai, T. 1988, ApJ, 328, 860.

Bai, T. and Sturrock, P.A. 1991, Nature, 350,141.

Bai, T. 1988, ApJ, 388, L69.

Bogart, R.S. 1982, Sol.Phys., 76, 155.

Brouwer, M.P. and Zwaan, C. 1990, Sol. Phys., 129, 221.

Bumba, V. and Howard, R. 1965, ApJ, 141, 1502.

Bumba, V. and Howard, R. 1969, Sol. Phys.,7, 28.

Carbonell, M. and Ballester, J.L. 1992, $A$ \& A, 235,350.

Carrington, R.C. 1855, M.N.R.A.S., 15, 174.

Castenmiller, M.J.M., Zwaan, C., and van der Zalm, E.B.J. 1986, Sol. Phys., $105,237$.

Dicke, R.H. 1976, Sol. Phys., 47, 475.

Gaizauskas, V., Harvey, K.L., Harvey, J.W., Zwaan, C. 1983, ApJ, 265, 1056

Goode, P.R. and Thompson, M.J. 1992, in The Solar Cycle, ed. K.L. Harvey,

NSO/Sacramento Peak 12th Summer Workshop, p. 182.

Knight, J.W., Schatten, K.H., and Sturrock, P.A. 1979, ApJ, 227, L153.

Leighton, R.B. 1964, ApJ, 140, 1547.

Parker, E.N. 1987, ApJ, 312, 868.

Petrovay, K. and Abuzeid, B.K. 1991, Sol. Phys., 131,231.

Rieger. E., Share, G.H., Forrest, D.J., Kanbach, G., Reppin, C., and Chupp,

E.L. 1984, Nature, 312, 623.

Sturrock, P.A. and Bai, T.1992, ApJ, 397, 337.

Svestka, Z. 1976, Solar Flares, D. Reidel, Dordrecht.

Wentzel, D. G. and Seiden, P.E. 1992, ApJ, 390, 280.

Wolff, C. 1983, ApJ, 264, 667, 